\title{
Common Bile Duct Polyp-Rare But Not So Rare Cause Of Obstructive Jaundice
}

\author{
Mayank Kabrawala ${ }^{1}$ Pankaj Desai ${ }^{2}$ Rajiv Mehta ${ }^{1}$ Subhash Nandwani ${ }^{1}$ Priya Arora ${ }^{3}$ \\ Chintan Patel $^{2}$ Keyur Bhatt ${ }^{4}$ Dhaval Mangukiya ${ }^{4} \quad$ Krishna Parekh $^{5}$
}

${ }^{1}$ Department of Gastroenterology, SIDS Hospital and

Research Center, Surat, Gujarat, India

${ }^{2}$ Department of Endoscopy and Endosonography, SIDS Hospital

and Research Center, Surat, Gujarat, India

3 Department of Histopathology, SIDS Hospital and Research Center,

Surat, Gujarat, India

${ }^{4}$ Department of Gastrointestinal and Minimal Access Surgery,

SIDS Hospital and Research Centre, Surat, Gujarat, India

${ }^{5}$ Department of Clinical Research, SIDS Hospital and

Research Center, Surat, Gujarat, India

\begin{abstract}
Address for correspondence Mayank Kabrawala, MD, DM, Department of Gastroenterology, SIDS Hospital and Research center, Majura Gate, Ring Road, Surat 395002 Gujarat, India (e-mail: mayankkabrawala@hotmail.com).
\end{abstract}

J Digest Endosc:2020;11:283-286

\begin{abstract}
Keywords

- common bile duct polyps

- obstructive jaundice

- ERCP

- sphincterotomy

Polyps are usually found in gastrointestinal tract as their primary site of occurrence. In contrast, polyps in common bile duct (CBD) are uncommon. Biliary polyps can manifest as obstructive jaundice, which is the usually a presentation of common bile duct stone or malignant tumor of bile duct and pancreas. In this case series, we present five patients who had clinical manifestations of obstructive jaundice. However, systematic diagnostic approach in these patients revealed presence of polyps in distal CBD. We have focused on diagnostic challenges encountered during the investigation of CBD polyps.
\end{abstract}

\section{Introduction}

Polyps are elevated growth from the mucosa ${ }^{1}$ and the common bile duct (CBD) polyps are an unusual disease entity. Clinical presentation of obstructive jaundice is usually due to CBD obstruction, which is most commonly due to CBD stone or malignant tumor. ${ }^{2}$ Other causes include benign biliary stricture, parasites, primary sclerosing cholangitis, AIDS-related cholangiopathy, and biliary tuberculosis.

It poses a challenge to diagnose a CBD polyp on the basis of abdominal ultrasound, contrast-enhanced computed tomography (CECT) abdomen, or magnetic resonance cholangiopancreatography (MRCP); however, endoscopic ultrasound (EUS) emerges as a good diagnostic modality, especially for distal CBD polyps. ${ }^{1,2}$ There is no consensus related to the treatment of CBD polyps. ${ }^{2}$ Hence, the patients are occasionally treated with the unnecessary extensive surgical procedure. ${ }^{1,2}$ Even though the CBD polyps are extremely rare; an awareness for such a rare entity is required as their potential existence may be confused between a malignant lesion and choledocholithiasis. Herein, we present a case-series of five adult male patients who presented with obstructive jaundice but eventually diagnosed with distal CBD polyps.

\section{Case Reports}

We examined five cases of CBD polyps retrospectively. The patient's age ranged from 60 to 82 years. The patients presented with obstructive jaundice. - Table 1 summarizes the clinical characteristics of the patients. On systematic evaluation, the CBD polyp was diagnosed ( - Fig. 1A-C). In all the patients, endoscopic retrograde cholangiopancreatography (ERCP) with biliary sphincterotomy was performed to retrieve polyps. Depending upon size of the polyps, small snare polypectomy or biopsy forceps was used ( - Fig. 1D, E). Macroscopic examination of resected polyps revealed sessile morphology (size varied from 10 to $21 \mathrm{~mm}$ ). Histopathological examinations showed tubulovillous adenoma in three patients
DOI https://doi.org/ 10.1055/s-0040-1713711 ISSN 0976-5042.
(C) 2020. Society of Gastrointestinal Endoscopy of India.

This is an open access article published by Thieme under the terms of the Creative Commons Attribution-NonDerivative-NonCommercial-License, permitting copying and reproduction so long as the original work is given appropriate credit. Contents may not be used for commercial purposes, or adapted, remixed, transformed or built upon. (https://creativecommons.org/licenses/by-nc-nd/4.0/).

Thieme Medical and Scientific Publishers Pvt. Ltd. A-12, 2nd Floor, Sector 2, Noida-201301 UP, India 
Table 1 Clinical presentation and histopathological characteristics of patients

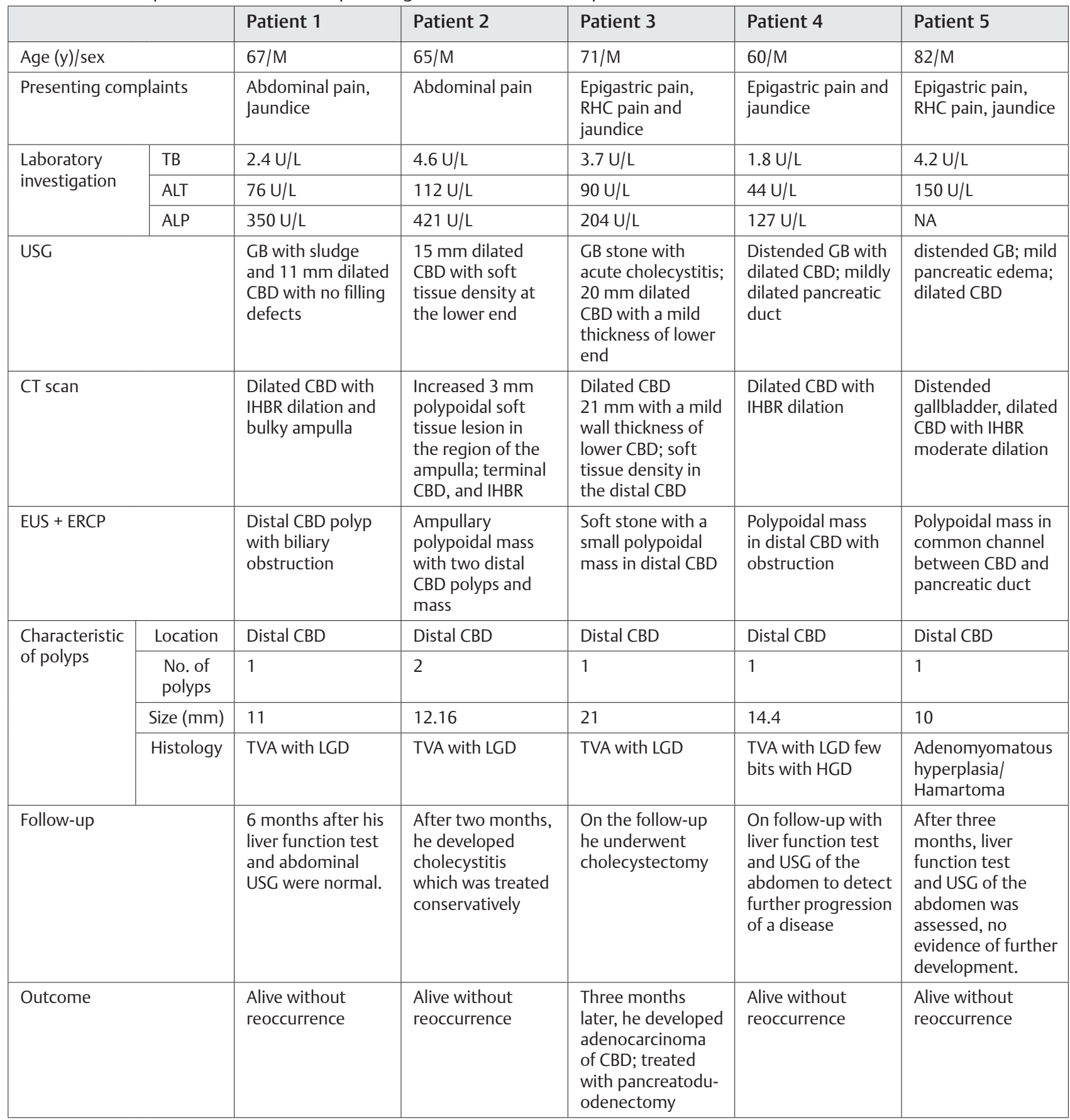

Abbreviations: CBD, common bile duct; ERCP, endoscopic retrograde cholangiopancreatography; EUS, endoscopic ultrasound; GB, gallbladder; HGD, high-grade dysplasia; IHBR, intrahepatic biliary radicals; LGD, low-grade dysplasia; M, male; NA, not available; TVA, tubulovillous adenoma; USG, ultrasonography; RHC, right hypochondrial; TB, total bilirubin; ALT, alanine tansferese; ALP, alanine phosphatase.

( $n=4$ with low-grade dysplasia and $n=1$ high-grade dysplasia [HGD]) and adenomyomatous hyperplasia in one patient (-Fig. 2B and C). All patients recovered uneventfully and are alive without recurrence except one patient (patient 3) who developed adenocarcinoma of CBD at 3-month follow-up (-Fig. 2A) and was successfully treated with pancreatoduodenectomy. The patient 4 , who had tubulovillous adenoma (TVA) with occasional focus of HGD on histopathology, was advised to undergo pancreaticoduodenectomy. However, the patient refused to undergo surgery. Hence, they were advised close follow-up with liver function test and ultrasonography in every 3 months and EUS at 6 months interval. We had follow-up available for 1 year and till then he did not have any evidence of recurrence of lesion.

\section{Discussion}

In 1988, Saxe et al was the first to narrate a case of CBD polyp with the histological characteristic of villous adenoma. Till the date, a total 39 cases have been reported in English 


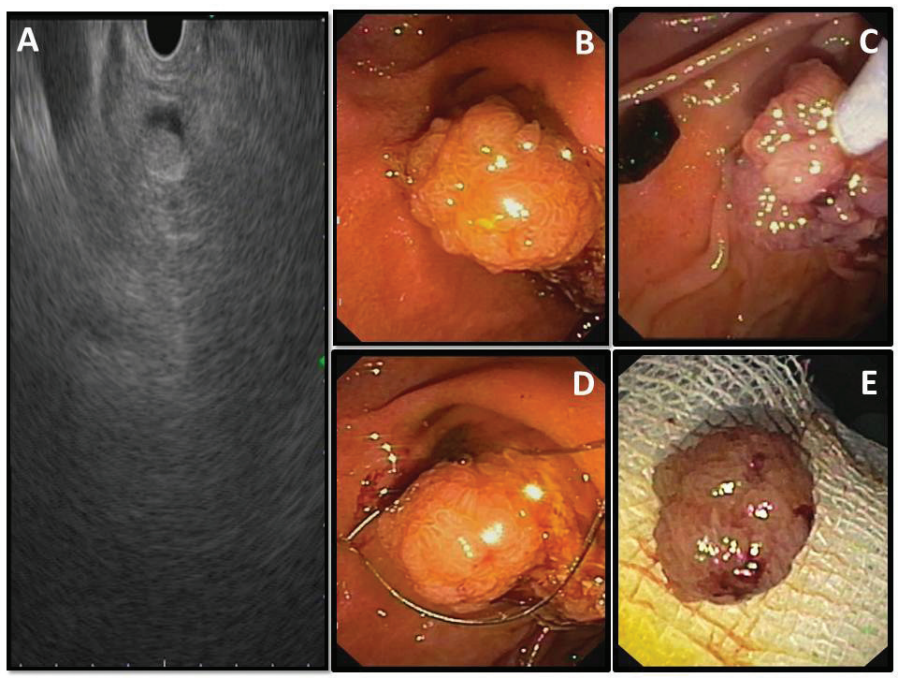

Fig. 1 Polypoid lesion and its resection within the distal CBD during endoscopic evaluation: (A) EUS examination of CBD polyp; (B) evaluation of distal CBD polyp with help of endosonography; (C) detection of distal CBD polyp; (D) resection of distal CBD polyp; (E) resected polyp. CBD, common bile duct; EUS, endoscopic ultrasound.

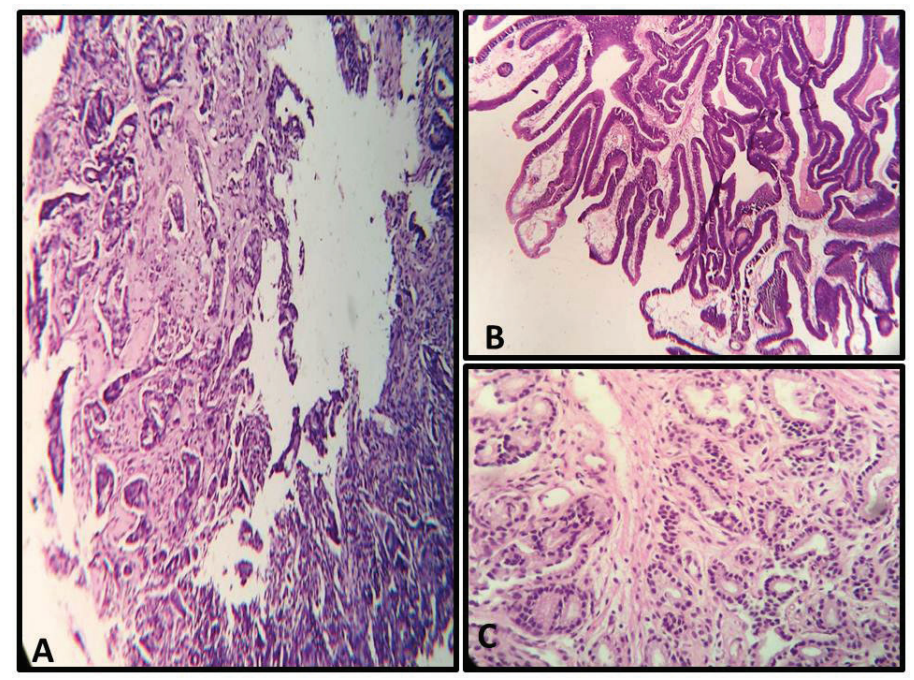

Fig. 2 Histopathological characteristics of adenomatous polyps of the common bile duct: (A) adenocarcinoma arising in tubulovillous adenoma; (B) tubulovillous adenoma with low-grade dysplasia; (C) adenomyomatous hyperplasia/hamartoma.

literature. Of these 39 cases, only few cases highlighted the diagnostic challenge of CBD polyp when the disease manifested with obstructive jaundice. We report, a case series of adult patients who presented with obstructive jaundice and subsequently diagnosed with CBD polyp.

Adenomas are benign tumors constituted from the glandular structure in epithelial tissues. They are generally found as single, well-defined polypoid lesion., ${ }^{2,3}$ TVA generally found in the gastrointestinal tract but uncommonly in the biliary system. ${ }^{2,45}$ In present case series, we identified four patients with tubulovillous adenoma and one patient with adenomyomatous hyperplasia. Although adenomatous polyps of extrahepatic bile duct and gallbladder are more commonly found in females, its predominant occurrence in male patients has been reported. ${ }^{6}$ Similarly, in our case series, we found male preponderance. CBD polyps usually present with innumerable clinical manifestation of CBD obstruction, that is, obstructive jaundice, right upper quadrant abdominal pain, dyspepsia, nausea, and vomiting. These clinical manifestations may occur due to bile duct obstruction. In our case series, all patients presented with jaundice and right upper quadrant abdominal pain. Laboratory examination showed elevated levels of total bilirubin and liver enzymes. Such clinical presentation and laboratory examination is more frequently observed in all biliary lesions which are causing bile duct obstruction. Owing to rarity of the disease, CBD adenoma remains unfocused during differential diagnosis., ${ }^{2,4}$ CBD polyps are incidentally identified during other biliary tract surgery. Preoperative diagnosis of CBD polyp is very difficult. Radiologic imaging techniques, CECT abdomen or MRCP usually help in differentiating polyp from a stone in the CBD. ${ }^{4}$ 
Even though CBD polyp is rare clinical entity, it should be considered during differential diagnosis in a group of patients presented with obstructive jaundice. EUS is a good diagnostic modality for differentiation in such scenario, especially for lower CBD lesions. Yusuf and Bhutani reported the negative predictive value of EUS is $97 \%$ for detection of CBD stone which is far better than ultrasonography (56\%) and CT scan (78\%). ${ }^{7}$ Another study by Choi et al diagnosed 132 patients with gallbladder polyp using EUS modality and also reported the sensitivity and specificity of $84.6 \%{ }^{8}$ Similarly, in the present case series, using EUS polyps were diagnosed in distal bile duct, just proximal to the ampulla which further confirmed the diagnosis of CBD polyps. We found EUS as the advantageous, reliable, and noninvasive diagnostic modality to differentiate CBD polyps from CBD stone and malignancy as compared with other noninvasive procedures. Histopathological examination is the only reliable method for final diagnosis of polyp or carcinoma. Tubular adenomas with morphological characteristics of TVA have a high risk of malignancy. , $^{2,9}$

An appropriate management approach of CBD polyp has not been clearly established as the entity is very rare. ${ }^{2}$ Sturgis et al were the first to implement the endoscopic resection of CBD polyp in the patient who had the high risk of malignancy. ${ }^{2,9}$ Likewise, local endoscopic polypectomy of the adenomatous polyp can be successfully performed. ${ }^{2}$ In our case series, all polyps were detected in the distal most part of CBD that made it more feasible to resect after a wide sphincterotomy using small snare polypectomy or biopsy forceps. Radical resection is advisable when malignancy is suspected or polyp size is more than $2 \mathrm{~cm} .{ }^{4}$ In cases of distal CBD carcinoma, pancreaticoduodenectomy should be considered as a mandatory treatment approach. We performed same in one of the patients who developed adenocarcinoma at follow-up. Ariche et al suggested that local resection with lymph node dissection of hepatoduodenal ligament might be curative in suspicious patients who have the high risk of a benign tumor. ${ }^{10}$ Several study reports prefer local resection for CBD adenoma, as they did not observe any complication or recurrence. But the major limitation of those case studies was, they reported less than 1 to 2 years to follow-up period. 2,4 The prognosis of CBD adenoma is good. But due to its anatomical site, it limits surgical approaches, which may lead to insufficient resection, as well as recurrence.

\section{Conclusion}

CBD bile duct polyp is an uncommon disease entity and prevailed with an enormously common clinical presentation. Such entity should be kept in mind during differential diagnosis of this region. EUS is an imperative tool for diagnosis of CBD polyp especially lower CBD. Histopathological examination is the gold-standard diagnosis, while endoscopic local resection can be considered as a treatment option for lower end CBD polyps.

\section{Conflict of Interest}

None declared.

\section{References}

1 Hacking CP, Taylor CJ, Nathanson LK. Common bile duct polyp mimicking choledocholithiasis: a case report with laparoscopic transcystic management. Surg Laparosc Endosc Percutan Tech 2008;18(3):290-293

2 Yusif-Zade K, Musayev J, Yeler M. Tubulopapillary adenoma of the common bile duct presenting with jaundice. Ulus Cerrahi Derg 2015;32(3):226-228

3 Saxe J, Lucas C, Ledgerwood AM, Sugawa C. Villous adenoma of the common bile duct. Arch Surg 1988;123(1):96

4 Čekas K, Rudaitis V, Beiša V, et al. Common bile duct villous adenoma: a case report and review of the literature. J Med Case Reports 2016;10(1):18

5 Loh KP, Nautsch D, Mueller J, Desilets D, Mehendiratta V. Adenomas involving the extrahepatic biliary tree are rare but have an aggressive clinical course. Endosc Int Open 2016;4(2):E112-E117

6 Kim B-S, Joo S-H, Joo K-R. Carcinoma in situ arising in a tubulovillous adenoma of the distal common bile duct: a case report. World J Gastroenterol 2008;14(29):4705-4708

7 Yusuf TE, Bhutani MS. Role of endoscopic ultrasonography in diseases of the extrahepatic biliary system. J Gastroenterol Hepatol 2004;19(3):243-250

8 Choi WB, Lee SK, Kim M, et al. A new strategy to predict the neoplastic polyps of the gallbladder based on a scoring system using EUS. Gastrointest Endosc 2000;52(3):372-379

9 Sturgis TM, Fromkes JJ, Marsh W Jr. Adenoma of the common bile duct: endoscopic diagnosis and resection. Gastrointest Endosc 1992;38(4):504-506

10 Ariche AM, Shelef I, Hilzenrat N, Dreznik Z. Villous adenoma of the common bile duct transforming into a cholangiocarcinoma. IMAJ-RAMAT GAN-. 2002 Dec 1;4(12):1149- 